\title{
A Method for Assaying Deubiquitinating Enzymes
}

\author{
Jae Il Lee, Seung Kyoon Woo, Keun Il Kim, Kyung Chan Park, Sung Hee Baek, Yung \\ Joon Yoo ${ }^{1}$, and Chin Ha Chung*
}

Department of Molecular Biology and Research Center for Cell Differentiation, College of Natural Sciences, Seoul National University, Seoul 151-742, Korea; ${ }^{1}$ Department of Life Science, Kwangju Institute of Science and Technology, Kwangju 506-303, Korea. *To whom correspondence should be addressed. E-mail: chchung@plaza.snu.ac.kr

\begin{abstract}
A general method for the assay of deubiquitinating enzymes was described in detail using ${ }^{125}$ I-labeled ubiquitin-fused $\alpha$ NH-MHISPPEPESEEEEEHYC (referred to as Ub-PESTc) as a substrate. Since the tyrosine residue in the PESTc portion of the fusion protein was almost exclusively radioiodinated under a mild labeling condition, such as using IODO-BEADS, the enzymes could be assayed directly by simple measurement of the radioactivity released into acid soluble products. Using this assay protocol, we could purify six deubiquitinating enzymes from chick skeletal muscle and yeast and compare their specific activities. Since the extracts of $E$. coli showed little or no activity against the substrate, the assay protocol should be useful for identification and purification of eukaryotic deubiquitinating enzymes cloned and expressed in the cells.
\end{abstract}

\section{INTRODUCTION}

Ubiquitin (Ub) is a highly conserved 76 amino acid protein found in all eukaryotic cells $(1,2)$. Ub is covalently ligated to a variety of intracellular proteins through an isopeptide linkage. Ubs by themselves or that have already been conjugated to proteins may also be ligated to additional Ub molecules to form branched poly-Ub chains. This ubiquitination has been implicated in the regulation of diverse cellular processes, such as selective protein breakdown, cell cycle regulation, and stress response (3-5). In addition, protein ubiquitination in vivo is a dynamic, reversible process that is under control responding to external stimuli, such as heat shock and starvation (6-8). Therefore, the enzymes that proteolytically remove $\mathrm{Ub}$ from $\mathrm{Ub}$-protein conjugates should be of importance in maintaining the steady-state levels of free $\mathrm{Ub}$ for its diverse cellular functions.

Ubs are encoded by two distinct gene classes. One is a poly-Ub gene that encodes a poly-protein of tandemly repeated Ubs $(9,10)$. The other encodes a fusion protein in which a single Ub is linked to a ribosomal protein consisting of 52 or 76-80 amino acids. The transient association of Ub with the ribosomal proteins has been suggested to promote their incorporation into ribosomes (11). Therefore, proteolysis at the peptide bonds between $\mathrm{Ub}$ and the extension proteins is required for generation of 
ribosomal proteins for ribosome biogenesis as well as of free Ubs.

Deubiquitinating enzymes (DUBs) are known to consist of a large protein family in eukaryotes. For example, the budding yeast has 17 genes for DUBs (4). Moreover, so far more than 60 full-length DUB sequences have been identified in eukaryotes (12). However, comprehensive searches for DUBs, particularly in mammalian cells, were hampered due to the lack of rapid and efficient methods for assaying the enzymes. We have recently reported that ${ }^{125}$ I-labeled Ub-PESTc serves as an excellent substrate for the sensitive and quantitative assay of various DUBs $(13,14)$. Using this assay, we have also isolated a number of novel DUBs in chick skeletal muscle and yeast $(13,15-18)$. Here we describe the detailed protocol for assaying DUBs using ${ }^{125}$ I-labeled Ub-PESTc. We also compare the sensitivity of this method to that using a fluorogenic peptide substrate, carbobenzoxy-LRGG-7-amido-4methylcoumarin (Cbz-LRGG-AMC), that has also been used as a substrate for DUBs (19).

\section{MATERIALS AND METHODS}

\section{Materials}

Yeast Ub hydrolase-1 (YUH1) was purified as described previously (20). The purified Ub-specific protease-6 (yUBP-6) in yeast and cUBP41, Ub C-terminal hydrolase-1 (cUCH-1), cUCH-6, and cUCH8 in chick skeletal muscle were prepared as described $(13,15-18)$. Ub-PESTc was purified from an $E$. coli strain AR13 carrying pNMHUB-PESTc as described by Yoo et al. (21). Ub-aldehyde was prepared as described (13). Cbz-LRGG-AMC was kindly provided by Dr. K. Tanaka (Tokyo Metropolitan Institute of Medical Science, Japan).

\section{Radioiodination of Ub-PESTc}

Ub-PESTc was radiolabeled with $\mathrm{Na}^{125} \mathrm{I}$ using IODO-BEADS (Pierce) by following the procedure recommended by the manufacturer and Markwell (22). One IODO-BEAD was incubated in $0.2 \mathrm{ml}$ of $0.1 \mathrm{M}$ Tris- $\mathrm{HCl}(\mathrm{pH} 7)$ in a microfuge tube for $5 \mathrm{~min}$ at room temperature. After incubation, $0.2 \mathrm{mg}$ of the purified Ub-PESTc and $200 \mu \mathrm{Ci}$ of $\mathrm{Na}^{125}$ I were added to the tube and further incubated for the next $15 \mathrm{~min}$. The radioiodinated Ub-PESTc (i.e., the liquid portion) was then removed and subjected to gel filtration on a Sephadex G-10 column equilibrated with the Tris buffer to remove free iodine. Upon the iodination procedure, approximately $85 \%$ of ${ }^{125} \mathrm{I}$ was incorporated into Ub-PESTc.

\section{Assay for hydrolysis of ${ }^{125}$ I-labeled Ub-PESTc}

Reaction mixtures (final $0.1 \mathrm{ml}$ ) contained proper amounts of the purified DUBs, $10-20 \mu \mathrm{g}$ of ${ }^{125} \mathrm{I}$ labeled Ub-PESTc, 0.1 M Tris-HCl (pH 7.8), 1 mM EDTA, 1 mM dithiothreitol, and 5\% (v/v) glycerol. After incubation of the mixtures for appropriate periods at $37^{\circ} \mathrm{C}$, the reaction was terminated by adding $50 \mu \mathrm{l}$ of $40 \%(\mathrm{v} / \mathrm{v})$ trichloroacetic acid and $50 \mu \mathrm{l}$ of $1.2 \%(\mathrm{w} / \mathrm{v})$ bovine serum albumin. The samples were vortexed and centrifuged for $10 \mathrm{~min}$ at $10,000 \mathrm{x}$ g using a microfuge, and aliquots $(0.1 \mathrm{ml})$ of the resulting supernatants were counted for radioactivity using a gamma-counter (13). 


\section{Assay for hydrolysis of Cbz-LRGG-AMC}

Reaction mixtures (final $0.1 \mathrm{ml}$ ) contained appropriate amounts of the purified DUBs, $0.2 \mathrm{mM} \mathrm{Cbz-}$ LRGG-AMC, $0.1 \mathrm{M}$ Tris-HCl (pH 7.8), $1 \mathrm{mM}$ EDTA, $1 \mathrm{mM}$ dithiothreitol, and 5\% (v/v) glycerol. After incubation of the mixtures for various periods at $37{ }^{\circ} \mathrm{C}$, the reaction was terminated by adding 0.1 $\mathrm{ml}$ of $1 \%$ (w/v) SDS and $0.8 \mathrm{ml}$ of $\mathrm{H}_{2} \mathrm{O}$. Release of free AMC by the enzyme reaction was determined by measuring its fluorescence at $380 \mathrm{~nm}$ (excitation) and $440 \mathrm{~nm}$ (emission) (23). Proteins were quantified as described by Bradford (24).

\section{Gel electrophoresis}

Polyacrylamide gel electrophoresis in the presence of SDS and 2-mercaptoethanol was performed using Tris-Tricine buffer as described by Schägger and von Jagow (25). The discontinuous slab gels contained 4, 10 and $16 \%$ polyacrylamide to improve resolution of small proteins. The sample buffer contained $150 \mathrm{mM}$ Tris- $\mathrm{HCl}(\mathrm{pH} 6.8), 1.5 \%$ (w/v) SDS, 2\% (v/v) 2-mercaptoethanol, 0.002\% (w/v) bromophenol blue and $7 \%(\mathrm{v} / \mathrm{v})$ glycerol. After electrophoresis, the gels were stained with Coomassie blue R-250 or subjected to autoradiography.

\section{RESULTS AND DISCUSSION}

Rechsteiner and coworkers (21) have constructed Ub- $\alpha$ NH-peptide extensions containing "PEST" sequences. Of these, Ub-PESTc contains a peptide extension of 18 amino acids, which carries a single tyrosine residue that can be radioiodinated and is short enough to be released as an acid-soluble product when incubated with DUBs. Moreover, they have reported that the recombinant Ub-PESTc protein can be easily purified by heating, such as at $85^{\circ} \mathrm{C}$, because fusion of the short peptide to Ub does not alter the heat resistance of $\mathrm{Ub}$ molecule. In addition, they have demonstrated that Ub-PESTc appears correctly processed to yield free $\mathrm{Ub}$ upon incubation with the chromatographic fractions of rabbit reticulocytes. Therefore, we chose Ub-PESTc for labeling with ${ }^{125} \mathrm{I}$ and hence for using the labeled protein as a substrate for the assay of DUBs. Overall procedure for the enzyme assay is summarized in Fig. 1.

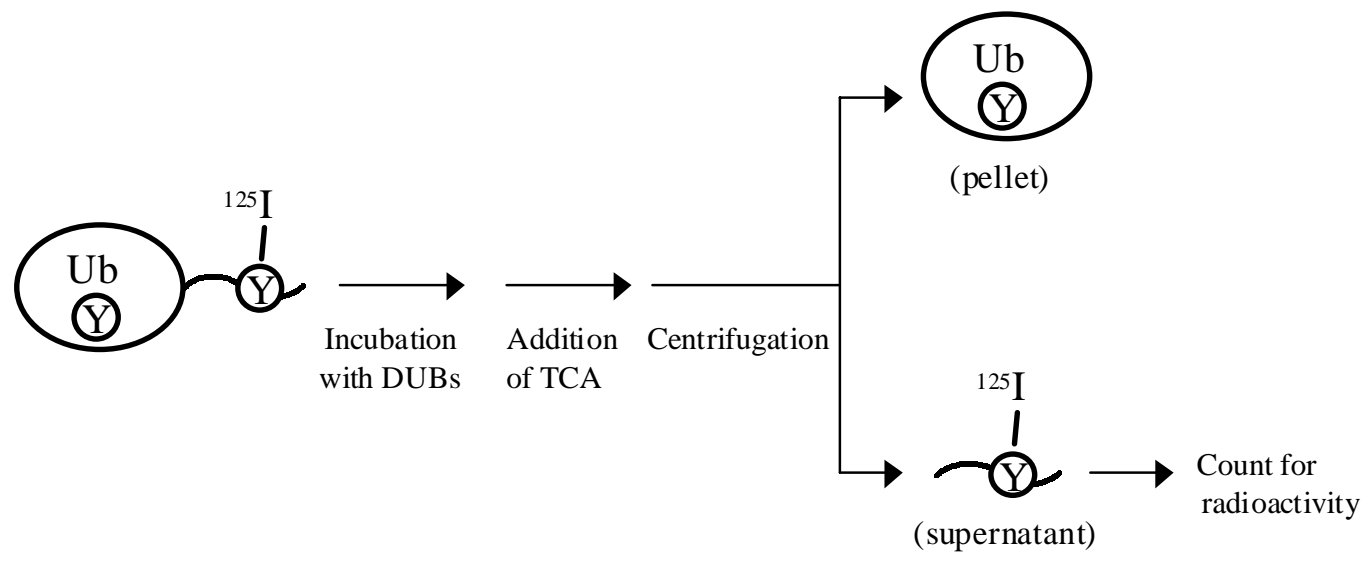

Fig. 1. Schematic representation for a method for assaying DUBs using ${ }^{125} \mathrm{I}-\mathrm{Ub}-\mathrm{PESTc}$. 
By following the protocol, we determined the activity of YUH1 by measuring its ability to release radioactive PESTc into an acid-soluble form from ${ }^{125}$ I-labeled Ub-PESTc. The purified enzyme was incubated with the radioiodinated substrate for various periods in the absence and presence of Ubaldehyde, which is known as a specific inhibitor of DUBs (26). Table 1 shows that the acid-soluble radioactivity increases in an incubation time-dependent fashion and this increase can be completely blocked by the treatment of Ub-aldehyde. Since YUH1 as well as other DUBs are known to specifically cleave the carboxyl side of the C-terminal Gly residue of Ub, the acid-soluble products should represent the PESTc peptide released from the Ub-peptide extension.

Table 1. Hydrolysis of ${ }^{125}$ I-labeled Ub-PESTc by the purified YUH1.

\begin{tabular}{ccc}
\hline $\begin{array}{c}\text { Incubation period } \\
(\text { min })\end{array}$ & $\begin{array}{l}\text { Radioactivity }(\mathrm{cpm}) \text { released into acid-soluble form } \\
\text { without }\end{array}$ & $\begin{array}{l}\text { with Ub-aldehyde } \\
0\end{array}$ \\
20 & 29 & 112 \\
40 & 1,235 & 147 \\
60 & 2,481 & 220 \\
\hline
\end{tabular}

\footnotetext{
${ }^{125}$ I-labeled Ub-PESTc $(20 \mu \mathrm{g})$ was incubated with YUH1 $(0.1 \mu \mathrm{g})$ at $37{ }^{\circ} \mathrm{C}$ for various periods in the presence and absence of $1 \mu \mathrm{g}$ of Ub-aldehyde. After incubation, radioactivity in the acid-soluble fraction was counted using a gamma-counter.
}

To validate further the assay method, ${ }^{125}$ I-labeled Ub-PESTc was incubated with increasing amounts of YUH1 for $30 \mathrm{~min}$ at $37^{\circ} \mathrm{C}$. The samples were then subjected to polyacrylamide gel electrophoresis in duplicate under denaturing conditions. After electrophoresis, one of the gels was stained with Coomassie R-250. Fig. 2A shows that the intensity of a protein band corresponding to the size of Ub increases upon incubation with increasing amounts of YUH1.

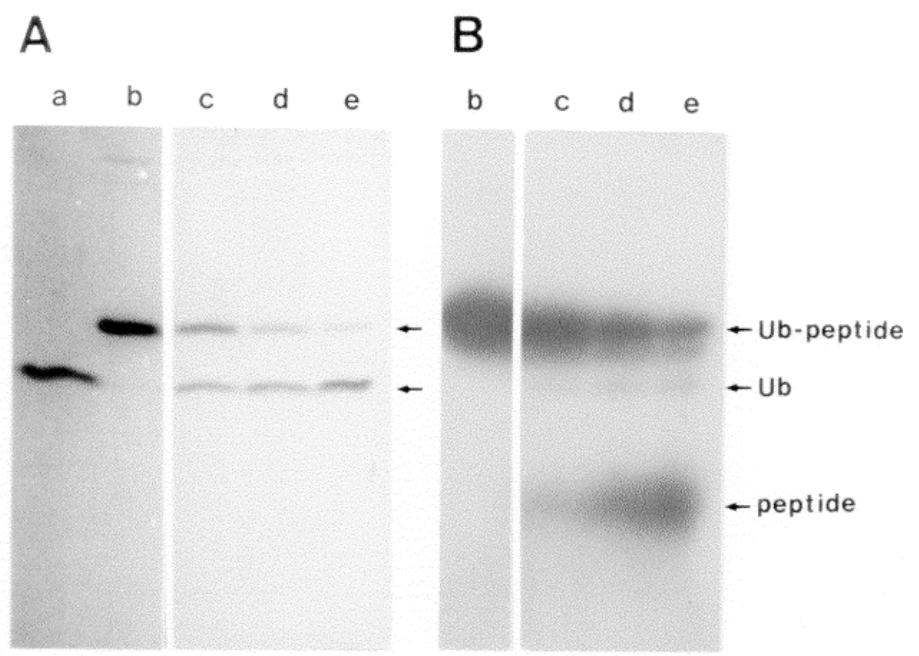

Fig. 2. SDS-polyacrylamide gel electrophoresis of the products generated by incubation of YUH1 and ${ }^{125} \mathrm{I}-$ labeled Ub-PESTc. The radioiodinated substrate $(10 \mu \mathrm{g})$ was incubated in the absence (lane b) and presence of 
$0.1 \mu \mathrm{g}$ (lane c), $0.2 \mu \mathrm{g}$ (lane d), and $0.4 \mu \mathrm{g}$ (lane e) of the purified YUH1 for $30 \mathrm{~min}$ at $37^{\circ} \mathrm{C}$. The samples were then electrophoresed in duplicate on a discontinuous gels containing SDS and 2-mercaptoethanol. After electrophoresis, one of the gels was stained with Coomassie R-250 (A), and the other was directly exposed on an X-ray film (B). Lane a contains $10 \mu \mathrm{g}$ of unlabeled $\mathrm{Ub}$ as a control.

In this gel, however, we could not find the band corresponding to the PESTc peptide, which might have been diffused out during the staining and destaining process. Therefore, the other gel was covered with a Saran wrap and directly exposed on an X-ray film. Upon the autoradiography, we were able to detect the band of the PESTc peptide, whose intensity also increased upon incubation with increasing amount of YUH1. In addition, this increase in the band intensity was approximately proportional to the increase in the release of acid-soluble radioactivity (data not shown). Furthermore, the amino acid sequence of the acid-soluble product determined by Edman degradation after separation from undigested ${ }^{125} \mathrm{I}-$ labeled Ub-PESTc by gel filtration was shown to be identical with the N-terminal sequence of PESTc (see Fig. 8 of ref. 13). Thus, it is clear that the acid-soluble radioactivity represents PESTc released from ${ }^{125}$ I-labeled PESTc by the action of YUH1.

Surprisingly, however, the band intensity of Ub upon the autoradiography was far lower than the others, despite the fact that $\mathrm{Ub}$ itself has a single tyrosine residue at the 59th position that can also be labeled by ${ }^{125} \mathrm{I}$. Typically, chloramine $\mathrm{T}$ is used for radioiodination of Ub molecules. In our studies, we used IODO-BEADS, in which chloramine $\mathrm{T}$ is immobilized on non-porous polystyrene beads. Perhaps, the tyrosine residue in $\mathrm{Ub}$ is not accessible to electrophilic idodine species (i.e., $\mathrm{I}^{+}$), which are produced by the immobilized chloramine $\mathrm{T}$, due to structural barrier, unlike that in PESTc, that is fused to the flexible Cterminal region of Ub. In any event, we could obtain ${ }^{125}$ I-labeled Ub-PESTc, in which PESTc was almost exclusively radioiodinated. This fortuitous finding allowed us to determine rapidly the activity of YUH1 as well as of other DUBs and to quantify precisely cleavage products by simple measurement of the radioactivity released into the acid-soluble products.

Using the assay method, we have previously purified several novel DUBs from chick skeletal muscle and yeast, including cUBP41 (15), cUCH-1 (16), cUCH-6 (13), cUCH-8 (17), and yUBP-6 (18), using conventional

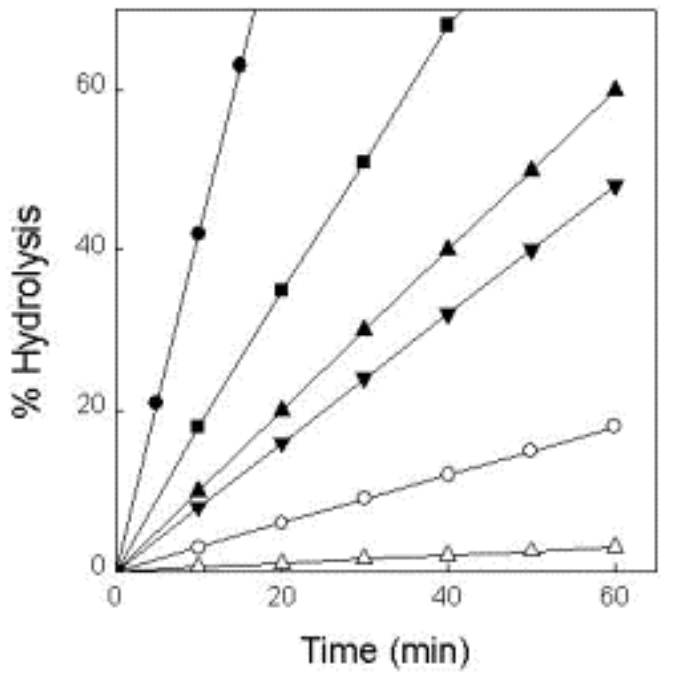

Fig. 3. Hydrolysis of ${ }^{125}$ I-labeled Ub-PESTc by various DUBs. The same amount $(0.1 \mu \mathrm{g})$ of the purified YUH1 (O), yUBP6 $(\Delta)$, cUCH-1 ( $)$, cUCH-6 ( ), cUCH-8 $(\boldsymbol{\Delta})$, or cUBP41 (ם) was incubated with $20 \mu \mathrm{g}$ of ${ }^{125} \mathrm{I}-\mathrm{Ub}$ PESTc at $37^{\circ} \mathrm{C}$ for various periods. After incubation, the release of PESTc was determined as described under Materials and Methods. chromatographic procedures. To compare the activities of these DUBs against ${ }^{125}$ I-Ub-PESTc, the same amount of each enzyme was incubated with the substrate for various periods. Fig. 3 shows that the hydrolytic rates differ markedly from each other. 
Among the DUBs, cUCH-6 hydrolyzed Ub-PESTc most rapidly. The $K_{\mathrm{m}}$ values for the enzymes were also different, ranging from 5 to $65 \mu \mathrm{M}$ (e.g., $5.1 \mu \mathrm{M}$ for cUCH-6 and $64.5 \mu \mathrm{M}$ for yUBP-6.)

$\mathrm{Ub}$ ends with the amino acid sequence of -RLRGG ${ }^{76}$. Based on the C-terminal sequence, Stein and coworkers (19) have synthesized a variety of fluorogenic peptides by conjugating AMC to the Ctermini of the peptides and used them as substrates for determination of the specificity of isopeptidase $\mathrm{T}$, which exists in all mammalian cells and hydrolyzes the isopeptide linkages of poly-Ub chains (27). Of these, Cbz-LRGG-AMC was used in the present studies to compare its sensitivity to the purified DUBs to that of ${ }^{125} \mathrm{I}-\mathrm{Ub}$-PESTc. Table 2 shows that all of the DUBs have at least three order higher specific activities against ${ }^{125} \mathrm{I}$-Ub-PESTc than those against Cbz-LRGG-AMC, indicating that the assay method using ${ }^{125} \mathrm{I}-\mathrm{Ub}$-PESTc is much more sensitive for determining the activities of DUBs than that using the fluorogenic peptide substrate. In addition, we have recently found that the extracts of $E$. coli by themselves are capable of releasing AMC from the peptide substrate (data not shown), implying that E. coli contains a protease(s), which specifically cleaves off AMC from the peptide substrate. On the other hand, the same extracts could not hydrolyze ${ }^{125} \mathrm{I}$-Ub-PESTc at all. Thus, the protease(s) is likely to interfere with the assay for overproduced eukaryotic DUBs in E. coli cells, when Cbz-LRGG-AMC was used as a substrate. Furthermore, extracts prepared from most of eukaryotic cells also contain a protease(s) that rapidly cleaves Cbz-LRGG-AMC but not ${ }^{125} \mathrm{I}-\mathrm{Ub}-\mathrm{PESTc}$. Thus, the assay method using ${ }^{125} \mathrm{I}$-Ub-PESTc should be appropriate for identification of DUBs in eukaryotic cells and their clones expressed in E. coli. In fact, we were able to identify and partially purify at least 10 different DUBs from the extract of chick skeletal muscle (13) and to purify several novel chick DUBs cloned and expressed in E. coli $(15,28)$.

Table 2. Comparison of the specific activities of various DUBs against Cbz-LRGG-AMC to those against ${ }^{125} \mathrm{I}-$ labeled Ub-PESTc.

\begin{tabular}{lcc}
\hline & \multicolumn{2}{c}{ Specific activity against } \\
DUBs & Cbz-LRGG-AMC & ${ }^{125} \mathrm{I}$-labeled Ub-PESTc \\
\hline YUH1 & $3.2 \times 10^{-10}$ & $5.1 \times 10^{-8}$ \\
yUBP6 & $2.0 \times 10^{-12}$ & $3.2 \times 10^{-9}$ \\
cUCH-1 & $0.7 \times 10^{-11}$ & $2.8 \times 10^{-7}$ \\
cUCH-6 & $1.2 \times 10^{-10}$ & $1.1 \times 10^{-6}$ \\
cUCH-8 & $0.9 \times 10^{-11}$ & $3.3 \times 10^{-7}$ \\
cUBP41 & $2.5 \times 10^{-11}$ & $5.3 \times 10^{-7}$ \\
\hline
\end{tabular}

The amounts of the enzymes used were $0.1 \mu \mathrm{g}$ and $5 \mu \mathrm{g}$ for assaying the hydrolysis of ${ }^{125} \mathrm{I}-\mathrm{Ub}-\mathrm{PESTc}$ and CbzLRGG-AMC, respectively. Incubations were performed at $37{ }^{\circ} \mathrm{C}$ for various periods to obtain initial velocity for each DUB. The specific activities of the enzymes were expressed as mol ${ }^{125}$ I-PESTc released into acid-soluble products or AMC released into the solution per min per mg protein.

Recently, Stein and coworkers (29) have developed a new assay method for DUBs based on the substrate Ub C-terminal AMC (Ub-AMC). They showed that the rate constants $\left(k_{\mathrm{c}} / K_{\mathrm{m}}\right)$ for the hydrolysis of Ub-AMC are $10^{4}$ - and $10^{7}$-fold over those for the cleavage of Cbz-LRGG-AMC for 
isopeptidase T andUCH-L3 respectively. UCH-L3 is a $26 \mathrm{kDa}$ Ub C-terminal hydrolase (30) isolated from rabbit reticulocytes. However, it has not yet been tested whether Ub-AMC is susceptible to any protease in bacterial or eukaryotic cells other than DUBs.

\section{ACKNOWLEDGMENTS}

We are grateful to Dr. Martin Rechsteiner (University of Utah) for providing E. coli strain AR13 carrying pNMHUB-PESTc, for which Ub-PESTc was purified. This work was supported by grants from Korea Science and Engineering foundation through Research Center for Cell Differentiation, Lotte Foundation, and Korea Ministry of Education (BSRI-97-4415).

\section{REFERENCES}

1. Rechsteiner, M. 1987. Ubiquitin-mediated pathways for intracellular proteolysis. Annu. Rev. Cell Biol. 3, 1-30.

2. Hershko, A., and Ciechanover, A. 1992. The ubiquitin system for protein degradation. Annu. Rev. Biochem. 61, 761-807.

3. Jentsch, S. 1992. The ubiquitin-conjugating system. Annu. Rev. Genet. 26, 179-207.

4. Hochstrasser, M. 1996. Ubiquitin-dependent protein degradation. Annu. Rev. Genet. 30, 405-439.

5. Wilkinson, K.D. 1995. Roles of ubiquitinylation in proteolysis and cellular regulation. Annu. Rev. Nutr. 15, 161-189.

6. Carlson, N., and Rechsteiner, M. 1987. Microinjection of ubiquitin: Intracellular distribution of and metabolism in HeLa cells maintained under normal physiological conditions. J. Cell Biol. 104, 537-546.

7. Carlson, N., Rogers, S., and Rechsteiner, M. 1987. Microinjection of ubiquitin: Changes in protein degradation in HeLa cells subjected to heat-shock. J. Cell Biol. 104, 547-555.

8. Haas, A.L. 1988. Immunochemical probes of Ub pool dynamics. In Ubiquitin (Rechsteiner, M., ed.) pp. 173-206, Plenum Press, New York.

9. Ozkaynak, E., Finley, D., and Varshavsky, A. 1984. The yeast ubiquitin gene: Head-to-tail repeats encoding a polyubiquitin precursor protein. Nature 312, 663-666.

10. Lund, P.K., Moasts-Staats, B.M., Simmons, J.G., Hoyt, E., D'Ercole, A.J., Martin, F., and Van Wyk, J.J. 1985. Nucleotide sequence analysis of a cDNA encoding human ubiquitin reveals that ubiquitin is synthesized as a precursor. J. Biol. Chem. 260, 7609-7613.

11. Finley, D., Bartel, B., and Varshavsky, A. 1989. The tails of ubiquitin precursors are ribosomal proteins whose fusion to ubiquitin facilitates ribosome biogenesis. Nature 338, 394-401.

12. Wilkinson, K.D. 1997. Regulation of ubiquitin-dependent processes by deubiquitinating enzymes. FASEB J. 11, 1245-1256.

13. Woo, S.K., Lee, J.I., Park, I.K., Yoo, Y.J., Cho, J.M., Tanaka, K., Kang, M.S., Ha, D.B. and Chung, C.H. 1995. Multiple ubiquitin C-terminal hydrolases from chick skeletal muscle. J. Biol. Chem. 270, 18766-18773.

14. Chung, C.H., Woo, S.K., Lee, J.I., Park, I.K., Kang, M.S., and Ha, D.B. 1996. Ubiquitin Cterminal hydrolases in chick skeletal muscle. Adv. Exp. Med. Biol. 384, 203-208. 
15. Baek, S.H., Choi, K.S., Yoo, Y.J., Cho, J.M., Baker, R.T., Tanaka, K., and Chung, C.H. 1997. Molecular cloning of a novel ubiquitin-specific protease, UBP41, with isopeptidase activity in chick skeletal muscle. J. Biol. Chem. 272, 25560-25565.

16. Woo, S.K., Baek, S.H., Lee, J.I., Yoo, Y.J., Cho, J.M., Kang, M.S., and Chung, C.H. 1997. Purification and characterization of a new ubiquitin C-terminal hydrolase (UCH-1) with isopeptidase activity from chick skeletal muscle. J. Biochem. 121, 684-689.

17. Baek, S.H., Woo, S.K., Lee, J.I., Yoo, Y.J., Cho, J.M., Kang, M.S., Tanaka, K., and Chung, C.H. 1997. New de-ubiquitinating enzyme, ubiquitin C-terminal hydrolase 8, in chick skeletal muscle. Biochem. J. 325, 325-330.

18. Park, K.C., Woo, S.K., Yoo, Y.J., Wyndham, A.M., Baker, R.T., and Chung, C.H. 1997. Purification and characterization of UBP6, a new ubiquitin specific protease in Saccharomyces cerevisiae. Arch. Biochem. Biophys. 347, 78-84.

19. Stein, R.L., Chen, Z., and Melandri, F. 1995. Kinetic studies of isopeptidase T: Modulation of peptidase activity by ubiquitin. Biochemistry 34, 12616-12623.

20. Miller, H.I., Henzel, W.J., Ridgway, J.B., Kuang, W.J., Chisholm, V., and Liu, C.C. 1989. Cloning and expression of a yeast ubiquitin-protein cleaving activity in E. coli. Bio/Technology 7, 698-704.

21. Yoo, Y., Rote, K., and Rechsteiner, M. 1989. Synthesis of peptides as cloned ubiquitin extensions. J. Biol. Chem. 264, 17078-17083.

22. Markwell, M.A.K. 1982. A new solid-phase reagent to iodinate proteins. Anal. Biochem. 125, $427-$ 435.

23. Yoo, S.J., Seol, J.H., Shin, D.H., Rohrwild, M., Kang, M.S., Tanaka, K., Goldberg, A.L., and Chung, C.H. 1996. Purification and characterization of the heat shock proteins HslV and HslU that forms a new ATP-dependent protease in Escherichia coli. J. Biol. Chem. 271, 14035-14040.

24. Bradford, M.M. A rapid and sensitive method of quantitation of microgram quantities of protein utilizing the principle of protein-dye binding. Anal. Biochem. 72, 248-254.

25. Schägger, H., and von Jagow, G. 1987. Tricine-sodium dodecyl sulfate polyacrylamide gel electrophoresis for the separation of proteins in the range from 1 to $100 \mathrm{kDa}$. Anal. Biochem. 166, 368-379.

26. Hershko, A., and Rose, I.A. 1977. Ubiquitin-aldehyde: a general inhibitor of ubiquitin-protein ligase system. Proc. Natl. Acad. Sci. U.S.A. 84, 1829-1833.

27. Wilkinson, K.D., Tashayev, V.L., O’Connor, L.B., Larsen, C.N., Kasperek, E., and Pickart, C.M. 1995. Metabolism of the polyubiquitin degradation signal: structure, mechanism, and role of isopeptidase T. Biochemistry 34, 14535-14546.

28. Baek, S.H., and Chung, C.H. manuscript in preparation.

29. Dang, L.C., Melandri, F.D., and Stein, R.L. 1998. Kinetic and mechanistic studies on the hydrolysis of ubiquitin C-terminal 7-amido-4-methylcoumarin by deubiquitinating enzymes. Biochemistry 37, 1868-1879.

30. Wilkinson, K.D., Deshpande, S., and Larsen, C.N. 1992. Comparisons of neuronal (PGP 9.5) and non-neuronal ubiquitin C-terminal hydrolases. Biochem. Soc. Trans. 20, 631-636. 